Proyecciones Journal of Mathematics

Vol. 36, No 2, pp. 209-224, June 2017.

Universidad Católica del Norte

Antofagasta - Chile

\title{
The total detour monophonic number of a graph
}

\author{
A. P. Santhakumaran \\ Hindustan Institute of Technology and Science, India \\ P. Titus \\ University College of Engineering Nagercoil, India \\ K. Ganesamoorthy \\ Coimbatore Institute of Technology, India \\ Received : December 2015. Accepted : January 2017
}

\begin{abstract}
For a connected graph $G=(V, E)$ of order at least two, a chord of a path $P$ is an edge joining two non-adjacent vertices of $P$. A path $P$ is called a monophonic path if it is a chordless path. A longest $x-y$ monophonic path is called an $x-y$ detour monophonic path. A set $S$ of vertices of $G$ is a detour monophonic set of $G$ if each vertex $v$ of $G$ lies on an $x-y$ detour monophonic path for some $x$ and $y$ in $S$. The minimum cardinality of a detour monophonic set of $G$ is the detour monophonic number of $G$ and is denoted by $d m(G)$. A total detour monophonic set of a graph $G$ is a detour monophonic set $S$ such that the subgraph induced by $S$ has no isolated vertices. The minimum cardinality of a total detour monophonic set of $G$ is the total detour monophonic number of $G$ and is denoted by $d m_{t}(G)$. A total detour monophonic set of cardinality $d m_{t}(G)$ is called a $d m_{t}$-set of $G$. We determine bounds for it and characterize graphs which realize the lower bound. It is shown that for positive integers $r, d$ and $k \geq 6$ with $r<d$ there exists a connected graph $G$ with monophonic radius $r$, monophonic diameter $d$ and $d m_{t}(G)=k$. For positive integers $a, b$ such that $4 \leq a \leq b$ with $b \leq 2 a$, there exists a connected graph $G$ such that $d m(G)=a$ and $d m_{t}(G)=b$. Also, if $p, d$ and $k$ are positive integers such that $2 \leq d \leq p-2,3 \leq k \leq p$ and $p-d-k+3 \geq 0$, there exists a connected graph $G$ of order p, monophonic diameter $d$ and $d m_{t}(G)=k$.
\end{abstract}

Key Words: Detour monophonic set, detour monophonic number, total detour monophonic set, total detour monophonic number.

AMS Subject Classification: 05C12. 


\section{Introduction}

By a graph $G=(V, E)$ we mean a finite undirected connected graph without loops and multiple edges. The order and size of $G$ are denoted by $p$ and $q$, respectively. For basic graph theoretic terminology we refer $[1,3]$. The distance $d(x, y)$ between two vertices $x$ and $y$ in a connected graph $G$ is the length of a shortest $x-y$ path in $G$. An $x-y$ path of length $d(x, y)$ is called an $x-y$ geodesic. The neighborhood of a vertex $v$ is the set $N(v)$ consisting of all vertices $u$ which are adjacent with $v$. A vertex $v$ is an extreme vertex if the subgraph induced by its neighbors is complete. Let $G_{1}=\left(V_{1}, E_{1}\right)$ and $G_{2}=\left(V_{2}, E_{2}\right)$ be two graphs with $V_{1} \cap V_{2}=\phi$, then the join $G_{1}+G_{2}$ is a graph $G=(V, E)$, where $V=V_{1} \cup V_{2}$ and $E=E_{1} \cup E_{2}$ together with all the edges joining vertices of $V_{1}$ to vertices of $V_{2}$ and $m_{j} K_{j}$ denotes $m_{j}$-copies of the complete graph $K_{j}$.

A chord of a path $P$ is an edge joining two non-adjacent vertices of $P$. A path $P$ is called a monophonic path if it is a chordless path. A longest $x-y$ monophonic path is called an $x-y$ detour monophonic path. A set $S$ of vertices of $G$ is a detour monophonic set of $G$ if each vertex $v$ of $G$ lies on an $x-y$ detour monophonic path for some $x$ and $y$ in $S$. The minimum cardinality of a detour monophonic set of $G$ is the detour monophonic number of $G$ and is denoted by $\operatorname{dm}(G)$. The detour monophonic number of a graph was introduced in [7] and further studied in [6].

A connected detour monophonic set of $G$ is a detour monophonic set $S$ such that the subgraph $G[S]$ induced by $S$ is connected. The minimum cardinality of a connected detour monophonic set of $G$ is the connected detour monophonic number of $G$ and is denoted by $d m_{c}(G)$. The connected detour monophonic number of a graph was introduced and studied in [8].

The detour monophonic concepts have interesting applications in Channel Assignment Problem in radio technologies. Also, there are useful applications of these concepts to security based communication network design.

For any two vertices $u$ and $v$ in a connected graph $G$, the monophonic distance $d_{m}(u, v)$ from $u$ to $v$ is defined as the length of a longest $u-v$ monophonic path in $G$. The monophonic eccentricity $e_{m}(v)$ of a vertex $v$ in $G$ is $e_{m}(v)=\max \left\{d_{m}(v, u): u \in V(G)\right\}$. The monophonic radius, $\operatorname{rad}_{m}(G)$ of $G$ is $\operatorname{rad}_{m}(G)=\min \left\{e_{m}(v): v \in V(G)\right\}$ and the monophonic diameter, $\operatorname{diam}_{m}(G)$ of $G$ is $\operatorname{diam}_{m}(G)=\max \left\{e_{m}(v): v \in V(G)\right\}$. The monophonic distance was introduced in [4] and further studied in [5].

The following theorems will be used in the sequel. 
Theorem 1.1. [7] Each extreme vertex of a connected graph $G$ belongs to every detour monophonic set of $G$. Moreover, if the set $S$ of all extreme vertices of $G$ is a detour monophonic set, then $S$ is the unique minimum detour monophonic set of $G$.

Theorem 1.2. [7] Let $G$ be a connected graph with a cut-vertex $v$ and let $S$ be a detour monophonic set of $G$. Then every component of $G-v$ contains an element of $S$.

Theorem 1.3. [8] Each extreme vertex of a connected graph $G$ belongs to every connected detour monophonic set of $G$.

Theorem 1.4. [8] Every cut-vertex of a connected graph $G$ belongs to every connected detour monophonic set of $G$.

Theorem 1.5. [8] For the complete graph $K_{p}(p \geq 2), d m_{c}\left(K_{p}\right)=p$.

Throughout this paper $G$ denotes a connected graph with at least two vertices.

\section{Total detour monophonic number}

Definition 2.1. A total detour monophonic set of a graph $G$ is a detour monophonic set $S$ such that the subgraph $G[S]$ induced by $S$ has no isolated vertices. The minimum cardinality of a total detour monophonic set of $G$ is the total detour monophonic number of $G$ and is denoted by $d m_{t}(G)$. A total detour monophonic set of cardinality $d m_{t}(G)$ is called a $d m_{t^{-}}$set of $G$.

Obviously, any connected detour monophonic set of $G$ is a total detour monophonic set of $G$.

Example 2.2. For the graph $G$ in Figure 2.1, it is clear that $S=\left\{v_{1}, v_{4}, v_{5}\right\}$ is a minimum detour monophonic set of $G$ so that $\operatorname{dm}(G)=3$. It is easily verified that the set $S_{1}=\left\{v_{1}, v_{2}, v_{4}, v_{5}\right\}$ is a minimum total detour monophonic set of $G$ so that $d m_{t}(G)=4$. Also, it is clear that $S_{2}=\left\{v_{1}, v_{2}, v_{3}, v_{4}, v_{5}\right\}$ is a minimum connected detour monophonic set of $G$ and so $d m_{c}(G)=5$. Thus the detour monophonic number, total detour monophonic number and connected detour monophonic number of a graph all different. 


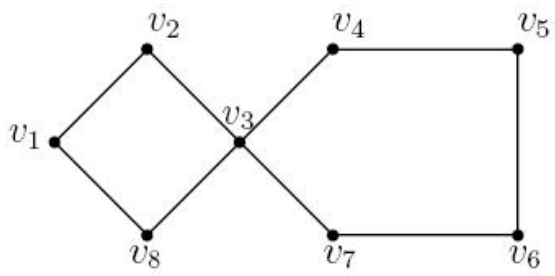

Figure 2.1: $G$

It was shown in [2] that determining the monophonic number of a graph is an NP-hard problem. Since every total detour monophonic set of $G$ is a monophonic set, determining the total detour monophonic number of a graph is also an NP-hard problem.

Definition 2.3. A vertex $v$ of a connected graph $G$ is called a support vertex of $G$ if it is adjacent to an end-vertex of $G$.

Theorem 2.4. Each extreme vertex and each support vertex of a connected graph $G$ belongs to every total detour monophonic set of $G$. If the set $S$ of all extreme vertices and support vertices form a total detour monophonic set, then it is the unique minimum total detour monophonic set of $G$.

Proof. Since every total detour monophonic set of $G$ is a detour monophonic set of $G$, by Theorem 1.1, each extreme vertex belongs to every total detour monophonic set of $G$. Since a total detour monophonic set of $G$ contains no isolated vertices, it follows that each support vertex of $G$ also belongs to every total detour monophonic set of $G$. Thus, if $S$ is the set of all extreme vertices and support vertices of $G$, then $d m_{t}(G) \geq|S|$. On the other hand, if $S$ is a total detour monophonic set of $G$, then $d m_{t}(G) \leq|S|$. Therefore $d m_{t}(G)=|S|$ and $S$ is the unique minimum total detour monophonic set of $G$.

Corollary 2.5. For the complete graph $K_{p}(p \geq 2), d m_{t}(G)=p$.

Theorem 2.6. Let $G$ be a connected graph with cut-vertices and let $S$ be a total detour monophonic set of $G$. If $v$ is a cut-vertex of $G$, then every component of $G-v$ contains an element of $S$. 
Proof. Since every total detour monophonic set of $G$ is a detour monophonic set of $G$, the result follows from Theorem 1.2.

Theorem 2.7. For a connected graph $G$ of order $p, 2 \leq d m(G) \leq d m_{t}(G) \leq$ $d m_{c}(G) \leq p$.

Proof. Any detour monophonic set of $G$ needs at least two vertices and so $d m(G) \geq 2$. Since every total detour monophonic set of $G$ is also a detour monophonic set of $G$, it follows that $d m(G) \leq d m_{t}(G)$. Also, since every connected detour monophonic set of $G$ is a total detour monophonic set of $G$, it follows that $d m_{t}(G) \leq d m_{c}(G)$. Since $V(G)$ is a connected detour monophonic set of $G$, it is clear that $d m_{c}(G) \leq p$. Hence $2 \leq d m(G) \leq$ $d m_{t}(G) \leq d m_{c}(G) \leq p$.

Corollary 2.8. Let $G$ be a connected graph. If $d m_{t}(G)=2$, then $d m(G)=$ 2 .

For any non-trivial path of order at least 4, the detour monophonic number is 2 and the total detour monophonic number is 4 . This shows that the converse of the Corollary 2.8 need not be true.

Remark 2.9. The bounds in Theorem 2.7 are sharp. For the complete graph $G=K_{2}, d m_{t}(G)=2$ and for the complete graph $K_{p}, d m_{t}\left(K_{p}\right)=p$. For the graph $G$ given in Figure 2.1, $d m(G)=3, d m_{t}(G)=4, d m_{c}(G)=5$ and $p=8$ so that $2<d m(G)<d m_{t}(G)<d m_{c}(G)<p$. Hence all the parameters in Theorem 2.7 are distinct.

Theorem 2.10. For any non-trivial tree $T$, the set of all end-vertices and support vertices of $T$ is the unique minimum total detour monophonic set of $G$.

Proof. Since the set of all end-vertices and support vertices of $T$ forms a total detour monophonic set, the result follows from Theorem 2.4.

Now we proceed to characterize graphs $G$ for which the lower bound in Theorem 2.7 is attained.

Theorem 2.11. For any connected graph $G, d m_{t}(G)=2$ if and only if $G=K_{2}$. 
Proof. If $G=K_{2}$, then $d m_{t}(G)=2$. Conversely, let $d m_{t}(G)=2$. Let $S=\{u, v\}$ be a minimum total detour monophonic set of $G$. Then $u v$ is an edge. It is clear that a vertex different from $u$ and $v$ cannot lie on a $u-v$ detour monophonic path and so $G=K_{2}$.

Theorem 2.12. Let $G$ be a connected graph with at least 2 vertices. Then $d m_{t}(G) \leq 2 d m(G)$.

Proof. Let $S=\left\{v_{1}, v_{2}, \ldots, v_{k}\right\}$ be a minimum detour monophonic set of $G$. Let $u_{i} \in N\left(v_{i}\right)$ for $i=1,2, \ldots, k$ and let $T=\left\{u_{1}, u_{2}, \ldots, u_{k}\right\}$. Then $S \cup T$ is a total detour monophonic set of $G$ so that $d m_{t}(G) \leq|S \cup T| \leq$ $2 k=2 d m(G)$.

Theorem 2.13. If $G=C_{p}$ or $G=\bar{K}_{2}+H(p \geq 3)$, where $H$ is a graph of order $p-2$, then $d m_{t}(G)=3$.

Proof. First, suppose that $G=C_{p}$. It is easily verified that any three consecutive vertices of $C_{p}$ is a minimum total detour monophonic set of $C_{p}$ and so $d m_{t}(G)=d m_{t}\left(C_{p}\right)=3$. Next, suppose that $G=\bar{K}_{2}+H$, where $H$ is a graph of order $p-2$. Let $V\left(\bar{K}_{2}\right)=\left\{u_{1}, u_{2}\right\}$. Then for any vertex $v$ of $H$, the set $S=\left\{v, u_{1}, u_{2}\right\}$ is a minimum total detour monophonic set of $G$ and so $d m_{t}(G)=3$.

Theorem 2.14. For the complete bipartite graph $G=K_{r, s}(2 \leq r \leq s)$,

$$
d m_{t}(G)= \begin{cases}3 & \text { if } 2=r \leq s \\ 4 & \text { if } 3 \leq r \leq s\end{cases}
$$

Proof. Let $U=\left\{u_{1}, u_{2}, \ldots, u_{r}\right\}$ and $W=\left\{w_{1}, w_{2}, \ldots, w_{s}\right\}$ be the bipartition of $G$. We prove this theorem by considering four cases.

Case 1. $2=r=s$. Then $G$ is the cycle $C_{4}$ and by Theorem 2.13, $d m_{t}(G)=3$.

Case 2. $2=r<s$. Then the minimum total detour monophonic set of $G$ is obtained by choosing the two elements from $U$ and any one element from $W$ and so $d m_{t}(G)=3$.

Case 3. $3 \leq r \leq s$. If $3=r=s$, then any minimum total detour monophonic set of $G$ is of the following forms: (i) $U \cup\left\{w_{j}\right\}$ for some $j(1 \leq j \leq 3),(i i) W \cup\left\{u_{i}\right\}$ for some $i(1 \leq i \leq 3)$, or $(i i i)$ a set containing any two elements from each of $U$ and $W$. If $3=r<s$, then any minimum total detour monophonic set of $G$ is either $U \cup\left\{w_{j}\right\}$ for some $j(1 \leq j \leq s)$, 
or any set containing any two elements from each of $U$ and $W$. Hence in both cases, we have $d m_{t}(G)=4$.

Case 4. $4 \leq r \leq s$. Then any minimum total detour monophonic set of $G$ contains any two elements from each of $U$ and $W$, and hence $d m_{t}(G)=4$.

Theorem 2.15. If $G=K_{1}+\bigcup m_{j} K_{j}$, where $j \geq 2, \sum m_{j} \geq 2$, then $d m_{t}(G)=p-1$.

Proof. Let $G=K_{1}+\bigcup m_{j} K_{j}$, where $j \geq 2$ and $\sum m_{j} \geq 2$. It is clear that $G$ has exactly one cut-vertex which is not the support vertex and all the remaining vertices are extreme vertices. Hence by Theorem 2.4, we have $d m_{t}(G)=p-1$.

\section{Some realization results on the total detour monophonic number}

For any connected graph $G, \operatorname{rad}_{m}(G) \leq \operatorname{diam}_{m}(G)$. It is shown in [4] that every two positive integers $a$ and $b$ with $a \leq b$ are realizable as the monophonic radius and monophonic diameter, respectively, of some connected graph. This theorem can also be extended so that the total detour monophonic number can be prescribed when $\operatorname{rad}_{m}(G)<\operatorname{diam}_{m}(G)$.

Theorem 3.1. For positive integers $r, d$ and $k \geq 6$ with $r<d$, there exists a connected graph $G$ such that $\operatorname{rad}_{m}(G)=r$, $\operatorname{diam}_{m}(G)=d$ and $d m_{t}(G)=k$.

Proof. We prove this theorem by considering two cases.

Case 1. $r=1$. Then $d \geq 2$. Let $C_{d+2}: v_{1}, v_{2}, \ldots, v_{d+2}, v_{1}$ be a cycle of order $d+2$. Let $G$ be the graph obtained by adding $k-3$ new vertices $u_{1}, u_{2}, \ldots, u_{k-3}$ to $C_{d+2}$ and joining each of the vertices

$u_{1}, u_{2}, \ldots, u_{k-3}, v_{3}, v_{4}, \ldots, v_{d+1}$ to the vertex $v_{1}$. The graph $G$ is shown in Figure 3.1. It is easily verified that $1 \leq e_{m}(x) \leq d$ for any vertex $x$ in $G$ and $e_{m}\left(v_{1}\right)=1, e_{m}\left(v_{2}\right)=d$. Then $\operatorname{rad}_{m}(G)=1$ and $\operatorname{diam}_{m}(G)=d$. 


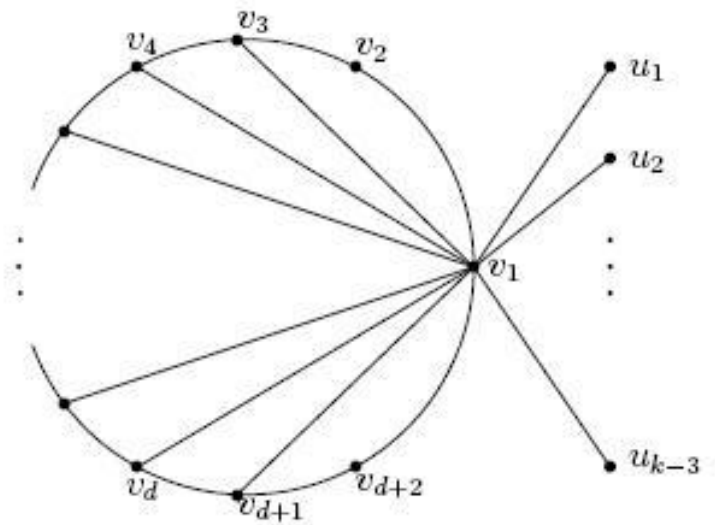

Figure 3.1: $G$

Let $S=\left\{u_{1}, u_{2}, \ldots, u_{k-3}, v_{2}, v_{d+2}, v_{1}\right\}$ be the set of all extreme vertices and support vertex of $G$. By Theorem 2.4, $S$ is a subset of any total detour monophonic set of $G$. It is clear that $S$ is the total detour monophonic set of $G$ and so $d m_{t}(G)=k$.

Case 2. $r \geq 2$. Let $C: v_{1}, v_{2}, \ldots, v_{r+2}, v_{1}$ be a cycle of order $r+2$ and let $W=K_{1}+C_{d+2}$ be the wheel with $V\left(C_{d+2}\right)=\left\{u_{1}, u_{2}, \ldots, u_{d+2}\right\}$. Let $H$ be the graph obtained from $C$ and $W$ by identifying $v_{1}$ of $C$ and the central vertex $K_{1}$ of $W$. If $d$ is odd, then add $k-6$ new vertices $w_{1}, w_{2}, \ldots, w_{k-6}$ to the graph $H$ and join each $w_{i}(1 \leq i \leq k-6)$ to the vertex $v_{1}$ and obtain the graph $G$ of Figure 3.2. It is easily verified that $r \leq e_{m}(x) \leq d$ for any vertex $x$ in $G$ and $e_{m}\left(v_{1}\right)=r$ and $e_{m}\left(u_{1}\right)=d$. Thus $\operatorname{rad}_{m}(G)=r$ and $\operatorname{diam}_{m}(G)=d$. Let $S=\left\{w_{1}, w_{2}, \ldots, w_{k-6}, v_{1}\right\}$ be the set of all extreme vertices and support vertex of $G$. By Theorem 2.4 , every total detour monophonic set of $G$ contains $S$. It is clear that $S$ is not a total detour monophonic set of $G$. Also, $S \cup\left\{x_{1}, x_{2}, x_{3}, x_{4}\right\}$ where $x_{j}(1 \leq j \leq 4) \in V(G)-S$, is not a total detour monophonic set of $G$. Let $T=S \cup\left\{u_{1}, u_{2}, u_{3}, v_{2}, v_{3}\right\}$. It is easily verified that $T$ is a total detour monophonic set of $G$ and so $d m_{t}(G)=k$. If $d$ is even, then add $k-5$ new vertices $w_{1}, w_{2}, \ldots, w_{k-5}$ to the graph $H$ and join each $w_{i}(1 \leq$ $i \leq k-5)$ to the vertex $v_{1}$ and obtain the graph $G$. Similar to the above argument, $T^{\prime}=\left\{w_{1}, w_{2}, \ldots, w_{k-5}, v_{1}, v_{2}, v_{3}, u_{1}, u_{\frac{d+4}{2}}\right\}$ is a minimum total detour monophonic set of $G$ and so $d m_{t}(G)=k$. 


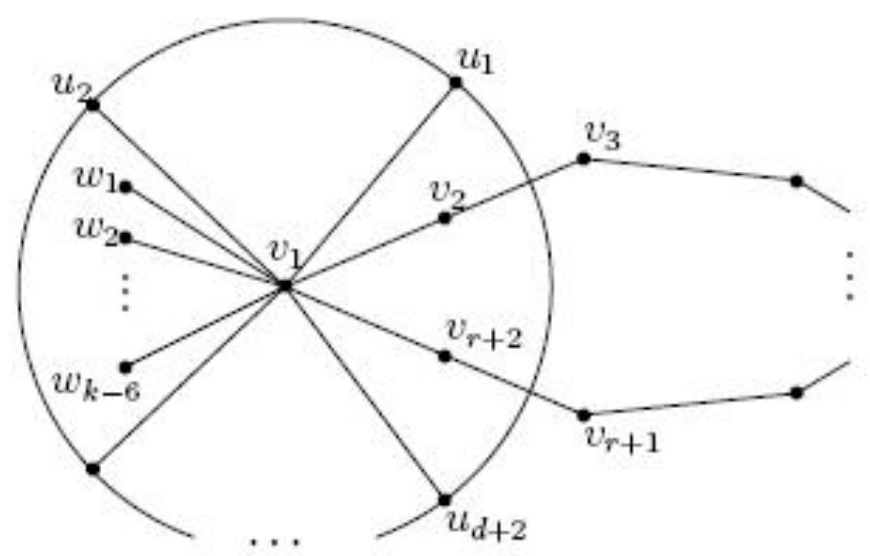

Figure 3.2: $G$

Problem 3.2. For any three positive integers $r, d$ and $k \geq 6$ with $r=d$ does there exist a connected graph $G$ with $\operatorname{rad}_{m}(G)=r$, $\operatorname{diam}_{m}(G)=d$ and $d m_{t}(G)=k$ ?

Theorem 3.3. If $p, d$ and $k$ are positive integers such that $2 \leq d \leq p-2$, $3 \leq k \leq p$ and $p-d-k+3 \geq 0$, then there exists a connected graph $G$ of order $p$, monophonic diameter $d$ and $d m_{t}(G)=k$.

Proof. We prove this theorem by considering two cases.

Case 1. $d=2$. First, let $k=3$. Let $P_{3}: v_{1}, v_{2}, v_{3}$ be the path of order 3. Now, add $p-3$ new vertices $w_{1}, w_{2}, \ldots, w_{p-3}$ to $P_{3}$. Let $G$ be the graph obtained by joining each $w_{i}(1 \leq i \leq p-3)$ to $v_{1}$ and $v_{3}$. The graph $G$ is shown in Figure 3.3. Then $G$ has order $p$ and monophonic diameter $d=2$. Clearly $S=\left\{v_{1}, v_{2}, v_{3}\right\}$ is a minimum total detour monophonic set of $G$ so that $d m_{t}(G)=k=3$. 


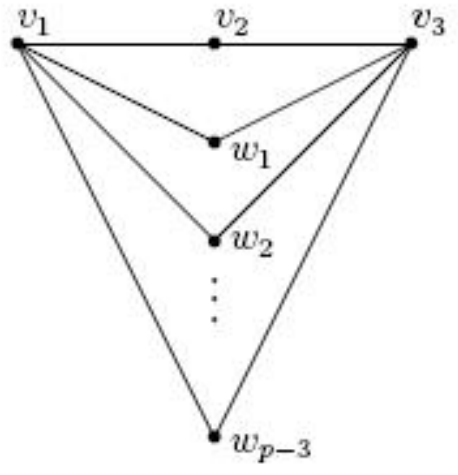

Figure 3.3: $G$

Now, let $4 \leq k \leq p$. Let $K_{p-1}$ be the complete graph with the vertex set $\left\{w_{1}, w_{2}, \ldots, w_{p-k+1}, v_{1}, v_{2}, \ldots, v_{k-2}\right\}$. Now, add the new vertex $x$ to $K_{p-1}$ and let $G$ be the graph obtained by joining $x$ with each vertex $w_{i}(1 \leq i \leq$ $p-k+1)$. The graph $G$ is shown in Figure 3.4. Then $G$ has order $p$ and monophonic diameter $d=2$. Let $S=\left\{v_{1}, v_{2}, \ldots v_{k-2}, x\right\}$ be the set of all extreme vertices of $G$. By Theorem 2.4, every total detour monophonic set of $G$ contains $S$. It is clear that $S$ is a detour monophonic set of $G$. Since the induced subgraph $G[S]$ has an isolated vertex, $d m_{t}(G) \geq k$. For any vertex $v \in\left\{w_{1}, w_{2}, \ldots, w_{p-k+1}\right\}$, it is clear that $S \cup\{v\}$ is a minimum total detour monophonic set of $G$ and so $d m_{t}(G)=k$.

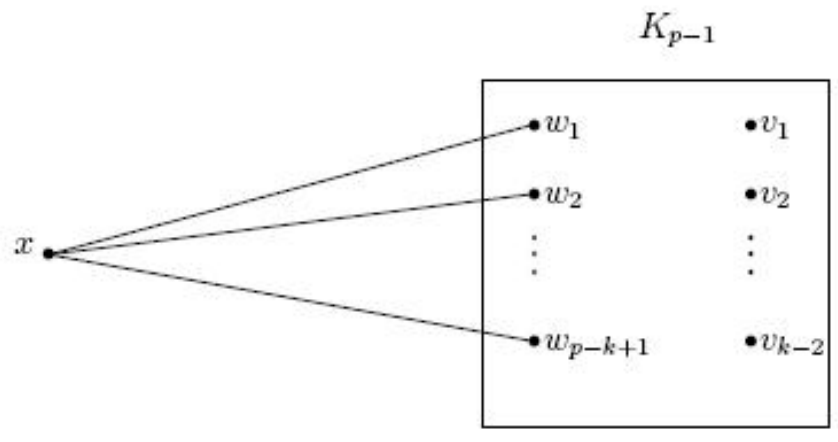

Figure 3.4: $G$

Case 2. $3 \leq d \leq p-2$. First, let $k=3$. Let $C_{d+2}: v_{1}, v_{2}, \ldots, v_{d+2}, v_{1}$ be the cycle of order $d+2$. Add $p-d-2$ new vertices $w_{1}, w_{2}, \ldots, w_{p-d-2}$ to $C$ and join each vertex $w_{i}(1 \leq i \leq p-d-2)$ to both $v_{1}$ and $v_{3}$, thereby producing 
the graph $G$ of Figure 3.5. Then $G$ has order $p$ and monophonic diameter d. It is clear that $S=\left\{v_{3}, v_{4}, v_{5}\right\}$ is a minimum total detour monophonic set of $G$ and so $d m_{t}(G)=3=k$.

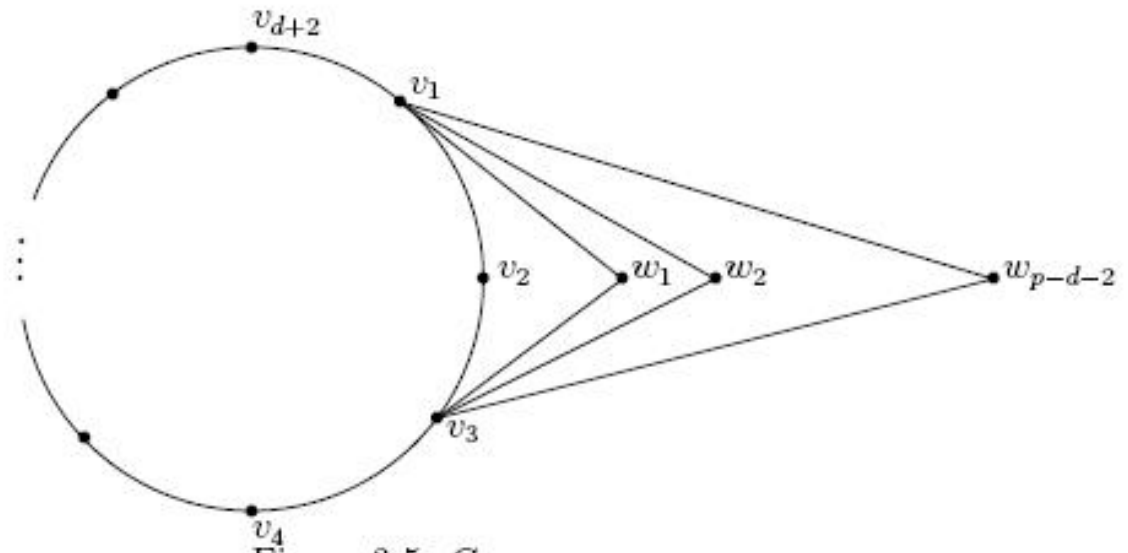

Figure 3.5: $G$

Now, let $k \geq 4$. Let $P_{d}: v_{0}, v_{1}, \ldots, v_{d-1}$ be a path of order $d$. Add $p-d$ new vertices $w_{1}, w_{2}, \ldots, w_{p-d-k+3}, u_{1}, u_{2}, \ldots, u_{k-3}$ to $P_{d}$ and join $w_{1}, w_{2}, \ldots, w_{p-d-k+3}$ to both $v_{0}$ and $v_{2}$; and join $u_{1}, u_{2}, \ldots, u_{k-3}$ to $v_{d-1}$, thereby producing the graph $G$ of Figure 3.6.
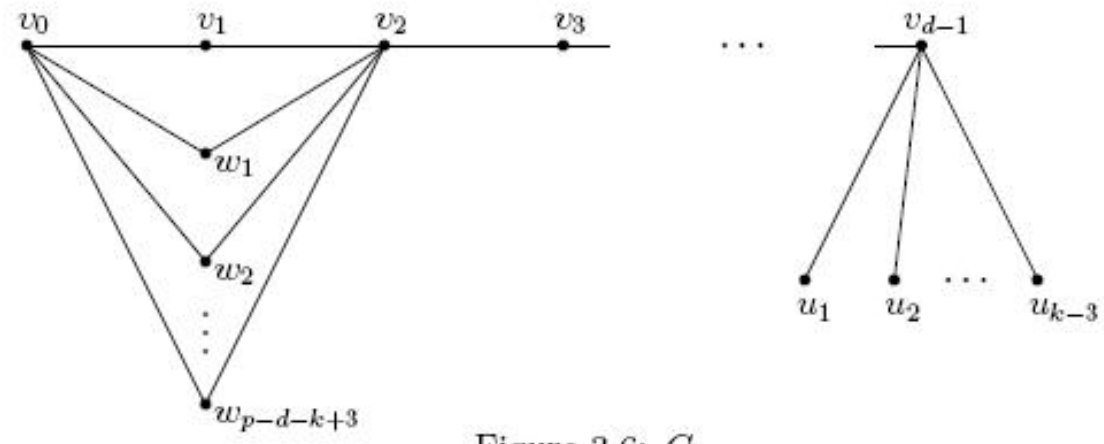

Figure 3.6: $G$

Then $G$ has order $p$ and monophonic diameter $d$. Let $S=\left\{u_{1}, u_{2}, \ldots, u_{k-3}\right.$, $\left.v_{d-1}\right\}$ be the set of all end-vertices and support vertex of $G$. By Theorem 2.4, every total detour monophonic set of $G$ contains $S$. It is clear that $S$ is not a total detour monophonic set of $G$. Also, for any $x \notin S, S \cup\{x\}$ is 
not a total detour monophonic set of $G$. It is easily seen that $S \cup\left\{v_{0}, v_{1}\right\}$ is a minimum total detour monophonic set of $G$ and so $d m_{t}(G)=k$.

In view of Theorems 2.13, 2.14 and 3.3, we leave the following problem as an open question.

Problem 3.4. Characterize graphs $G$ for which $d m_{t}(G)=3$.

In view of Theorem 2.7, we have the following realization result.

Theorem 3.5. If $a, b$ and $p$ are positive integers such that $4 \leq a \leq b \leq$ $p$, then there exists a connected graph $G$ of order $p, d m_{t}(G)=a$ and $d m_{c}(G)=b$.

Proof. We prove this theorem by considering four cases.

Case 1. $4 \leq a=b=p$. Let $G=K_{p}$. Then by Corollary 2.5 and Theorem 1.5 , we have $d m_{t}(G)=d m_{c}(G)=p$.

Case 2. $4 \leq a<b<p$. Let $P_{b-a+3}: u_{1}, u_{2}, \ldots, u_{b-a+3}$ be a path of order $b-a+3$. Add $p-b+a-3$ new vertices $v_{1}, v_{2}, \ldots, v_{p-b}, w_{1}, w_{2}, \ldots, w_{a-3}$ to $P_{b-a+3}$ and join $v_{1}, v_{2}, \ldots, v_{p-b}$ with both $u_{1}$ and $u_{3}$; and also join each of $w_{i}(1 \leq i \leq a-3)$ with $u_{b-a+3}$, thereby producing the graph $G$ of Figure 3.7. Then $G$ has order $p$. Let $S=\left\{w_{1}, w_{2}, \ldots, w_{a-3}, u_{b-a+3}\right\}$ be the set of all extreme vertices and support vertex of $G$. By Theorem 2.4, every total detour monophonic set of $G$ contains $S$. It is clear that for any $x \notin S, S \cup\{x\}$ is not a total detour monophonic set of $G$. Since $S \cup\left\{u_{1}, u_{2}\right\}$ is a total detour monophonic set of $G$, we have $d m_{t}(G)=a$.

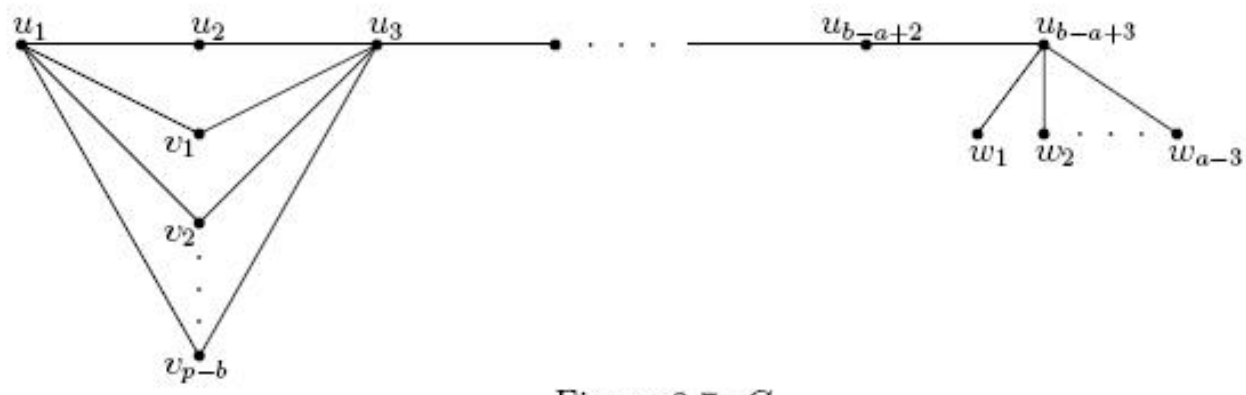

Figure 3.7: $G$

Let $S_{1}=\left\{w_{1}, w_{2}, \ldots, w_{a-3}, u_{3}, u_{4}, \ldots, u_{b-a+3}\right\}$ be the set of all extreme vertices and cut-vertices of $G$. By Theorems 1.3 and 1.4, every connected 
detour monophonic set of $G$ contains $S_{1}$. It is clear that for any $x \notin S_{1}, S_{1} \cup$ $\{x\}$ is not a connected detour monophonic set of $G$. Let $S_{1}^{\prime}=S_{1} \cup\left\{u_{1}, u_{2}\right\}$. It is easily verified that $S_{1}^{\prime}$ is a connected detour monophonic set of $G$ and so $d m_{c}(G)=b$.

Case 3. $4 \leq a=b<p$. Let $P_{3}: v_{1}, v_{2}, v_{3}$ be a path of order 3. Let $H$ be the graph obtained from $P_{3}$ by adding $p-a$ new vertices $w_{1}, w_{2}, \ldots, w_{p-a}$ to $P_{3}$ and join each $w_{i}(1 \leq i \leq p-a)$ to $v_{1}$ and $v_{3}$. Also, add $a-3$ new vertices $u_{1}, u_{2}, u_{3}, \ldots, u_{a-3}$ to $H$ and join each $u_{i}(1 \leq i \leq a-3)$ with $v_{2}$, thereby producing the graph $G$ in Figure 3.8 of order $p$. Let $S=\left\{u_{1}, u_{2}, \ldots, u_{a-3}, v_{2}\right\}$ be the set of all extreme vertices and support vertex of $G$. By Theorems 2.4, 1.3 and 1.4, every total detour monophonic set and every connected detour monophonic set of $G$ contains $S$. It is clear that for any $x \notin S, S \cup\{x\}$ is not a total detour monophonic set of $G$. Since $S \cup\left\{v_{1}, v_{3}\right\}$ is a connected detour monophonic set of $G$ and also a total detour monophonic set of $G$, we have $d m_{c}(G)=d m_{t}(G)=a$.

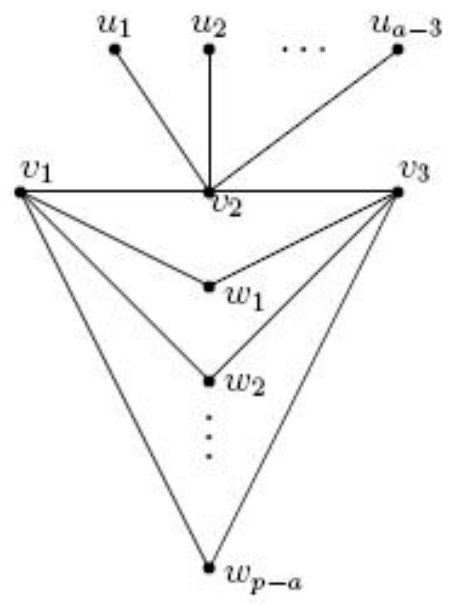

Figure 3.8: $G$

Case 4. $4 \leq a<b=p$. Let $P_{b-a+3}: u_{1}, u_{2}, \ldots, u_{b-a+3}$ be a path of order $b-a+3$. Add $a-3$ new vertices $v_{1}, v_{2}, \ldots, v_{a-3}$ to $P_{b-a+3}$ and join each $v_{i}(1 \leq i \leq a-3)$ with $u_{b-a+3}$, thereby producing the graph $G$ in Figure 3.9 of order $p=b$. Let $S=\left\{u_{1}, v_{1}, v_{2}, \ldots, v_{a-3}, u_{2}, u_{b-a+3}\right\}$ be the set of all extreme vertices and support vertices of $G$. By Theorem 2.4, every total detour monophonic set of $G$ contains $S$. It is clear that $S$ is the total detour monophonic set of $G$ and so $d m_{t}(G)=a$. 


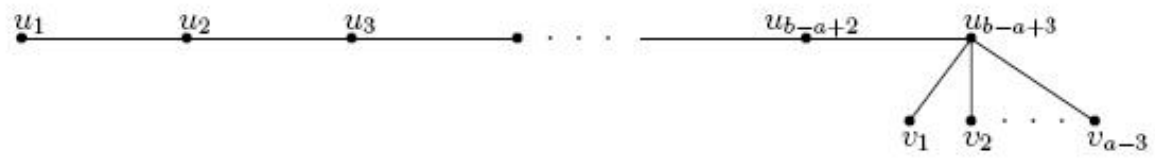

Figure 3.9: $G$

It is clear that $V(G)$ is the set of all extreme vertices and cut-vertices of $G$. Then by Theorems 1.3 and $1.4, V(G)$ is the connected detour monophonic set of $G$ and so $d m_{c}(G)=b=p$.

Theorem 3.6. For positive integers $a, b$ such that $4 \leq a \leq b$ with $b \leq 2 a$, there exists a connected graph $G$ such that $d m(G)=a$ and $d m_{t}(G)=b$.

Proof. Case 1. $4 \leq a=b$. Then the complete graph $K_{a}$ has the desired properties.

Case 2. $4 \leq a<b$. Let $b=a+k$, where $1 \leq k \leq a$. For $k=1$, the $\operatorname{star} K_{1, a}$ has the desired properties. Now, let $k \geq 2$. Let $C_{i}: x_{i}, y_{i}, z_{i}, w_{i}, x_{i}(1 \leq i \leq$ $k-1$ ) be " $k-1$ " copies of $C_{4}$. Let $H$ be the graph formed by identifying the vertices $x_{i}$ from $C_{i}(1 \leq i \leq k-1)$ and let $x$ be the identified vertex. Let $G$ be the graph obtained from $H$ by adding $a-k+1$ new vertices $v_{1}, v_{2}, \ldots, v_{a-k+1}$ and joining each $v_{i}(1 \leq i \leq a-k+1)$ with $x$. The graph $G$ is shown in Figure 3.10. Let $S=\left\{v_{1}, v_{2}, \ldots, v_{a-k+1}\right\}$ be the set of all extreme vertices of $G$. By Theorem 1.1, every detour monophonic set of $G$ contains $S$. It is clear that $S_{1}=S \cup\left\{z_{1}, z_{2}, \ldots, z_{k-1}\right\}$ is the detour monophonic set of $G$ and so $d m(G)=a$.

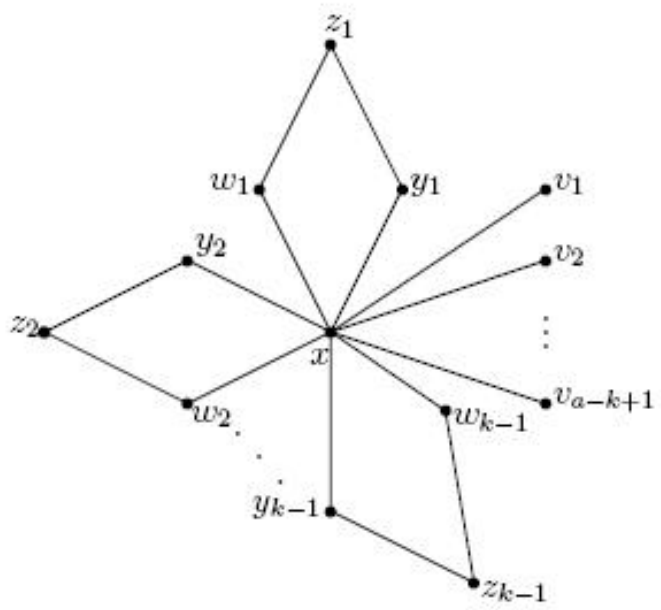

Figure 3.10: $G$ 
Let $S^{\prime}=S \cup\{x\}$ be the set of all extreme vertices and support vertex of $G$. By Theorem 2.4, every total detour monophonic set of $G$ contains $S^{\prime}$. Also, by Theorem 2.6, every total detour monophonic set of $G$ contains at least one vertex from each $V\left(C_{i}\right)-\{x\}$. Hence $S^{\prime \prime}=$ $S^{\prime} \cup\left\{y_{1}, z_{1}, y_{2}, z_{2}, \ldots, y_{k-1}, z_{k-1}\right\}$ is a minimum total detour monophonic set of $G$ so that $d m_{t}(G)=b$.

\section{References}

[1] F. Buckley and F. Harary, Distance in Graphs, Addison-Wesley, Redwood City, CA, (1990).

[2] M. C. Dourado, F. Protti and J.L. Szwarcfiter, Algorithmic Aspects of Monophonic Convexity, Electronic Notes in Discrete Mathematics, 30 (2008) 177-182.

[3] F. Harary, Graph Theory, Addison-Wesley, 1969.

[4] A. P. Santhakumaran and P. Titus, Monophonic Distance in Graphs, Discrete Mathematics, Algorithms and Applications, Vol. 3, No. 2, pp. 159-169, (2011).

[5] A. P. Santhakumaran and P. Titus, A Note on "Monophonic Distance in Graphs", Discrete Mathematics, Algorithms and Applications, Vol. 4, No. 2 (2012).

[6] P. Titus and K. Ganesamoorthy, On the Detour Monophonic Number of a Graph, Ars Combinatoria, 129, pp. 33-42, (2016).

[7] P. Titus, K. Ganesamoorthy and P. Balakrishnan, The Detour Monophonic Number of a Graph, J. Combin. Math. Combin. Comput., 84, pp. 179-188, (2013).

[8] P. Titus, A.P. Santhakumaran and K. Ganesamoorthy, The Connected Detour Monophonic Number of a Graph, TWMS Journal of Applied and Engineering Mathematics, Vol. 6, No.1, pp. 75-86, (2016). 


\section{A. P. Santhakumaran}

Department of Mathematics

Hindustan Institute of Technology and Science

Chennai - 603 103,

India

e-mail: apskumar1953@yahoo.co.in

\section{P. Titus}

Department of Mathematics,

University College of Engineering Nagercoil

Anna University: Tirunelveli Region

Nagercoil - 629 004,

India

e-mail: titusvino@yahoo.com

and

\section{K. Ganesamoorthy}

Department of Mathematics, Coimbatore Institute of Technology

Government Aided Autonomous Institution

Coimbatore - 641014 ,

India

e-mail: kvgm_2005@yahoo.co.in 